TITLE:

\title{
A quantum solute-solvent interaction using spectral representation technique applied to the electronic structure theory in solution
}

\author{
$\operatorname{AUTHOR}(\mathrm{S})$ : \\ Yamazaki, T; Sato, H; Hirata, F
}

\section{CITATION:}

Yamazaki, T ...[et al]. A quantum solute-solvent interaction using spectral representation technique applied to the electronic structure theory in solution. JOURNAL OF CHEMICAL PHYSICS 2003, 119(13): 6663-6670

ISSUE DATE:

2003-10-01

URL:

http://hdl.handle.net/2433/39754

\section{RIGHT:}

Copyright 2003 American Institute of Physics. This article may be downloaded for personal use only. Any other use requires prior permission of the author and the American Institute of Physics. 


\title{
A quantum solute-solvent interaction using spectral representation technique applied to the electronic structure theory in solution
}

\author{
Takeshi Yamazaki \\ Department of Theoretical Study, Institute for Molecular Science, Okazaki, Aichi 444-8585, Japan \\ Hirofumi Sato \\ Department of Molecular Engineering, Kyoto University, Sakyo-ku, Kyoto 606-8501, Japan \\ Fumio Hirata ${ }^{\text {a) }}$ \\ Department of Theoretical Study, Institute for Molecular Science, and Department of Functional Molecular \\ Science, The Graduate University for Advanced Studies, Okazaki, Aichi 444-8585, Japan
}

(Received 17 February 2003; accepted 7 July 2003)

\begin{abstract}
In this paper, we present a new approach to treat the electronic structure of a molecule in solution. Unlike the hybrid-type method, such as the reference interaction site model self-consistent-field theory, the new approach describes not only the electronic structure of solute but also solute-solvent interactions in terms of the quantum chemistry based on the Hartree-Fock frozen density formulation. In the treatment, the quantum effect due to solvent, including exchange repulsion, is projected on to the solute Hamiltonian using the spectral representation method. The solvent distribution around the solute is handled by the integral equation theory of liquids. As illustrative applications of the approach, the electronic and solvation structure of noble atoms, neon and argon, in liquid neon are studied. We also investigate the electronic structure of an excess electron in liquid helium. The preliminary results demonstrate that the quantum-mechanical effect on the electronic and solvation structure of the solute due to solvent molecules is successfully represented by the new method. (C) 2003 American Institute of Physics. [DOI: 10.1063/1.1604381]
\end{abstract}

\section{INTRODUCTION}

Elucidating solvation effect on molecules in solution is one of the most important issues in the area of chemistry, biology, and medicine, since most chemical processes of interest occur and are observed in solutions. Due to the latest development in the experimental techniques, such as the ultrafast spectroscopy, it has become possible to trace the quantum processes in molecules in liquid phases. It is natural that more and more quantum chemists have become interested in the electronic structure of molecules in solutions. Many theoretical methods have been proposed for treating chemical processes in solutions in the last two decades. ${ }^{1-3}$ The approach typical to most of those theories so far is a hybrid between the quantum and classical treatments applied, respectively, to solute and solvent, such as the reference interaction site model self-consistent-field (RISM-SCF) method, ${ }^{4-6}$ since it is impossible to solve the Schrödinger equation for an entire system including all the solvent molecules. The question how we connect the quantum solute and classical solvent in such hybrid theories is equivalent to asking how we describe the interaction between the solute and solvent. Most of those theories treat the interaction as a sum of the classical electrostatic interaction and classical shortrange interactions, such as the Lennard-Jones interaction. Consequently, the interaction does not include the quantummechanical effects explicitly.

Recently, attempts have been proposed to defeat the situ-

a)Electronic mail: hirata@ims.ac.jp ation in which solvents are represented classically. Amovilli and Mennucci $^{7}$ have derived an expression for the Pauli repulsion and dispersion contribution to the solvation free energy in terms of the polarizable continuum model. In the development of the so-called QM/MM methods, Gordon and co-workers ${ }^{8}$ have proposed the effective fragment potential (EFP) to treat solvent effects on chemical properties and reactions. The EFP simulates the important nonbonded energy terms, including Coulomb interactions, polarization, and exchange repulsion. Warshel et al. ${ }^{9}$ have proposed the frozen density functional theory, which is applied to ab initio freeenergy calculations of chemical reactions in solution. The potential of the solvent molecules exerted on the solute is derived from a formal DFT treatment by freezing the electronic density of solvents. More recently, Yoshida and Kato ${ }^{10}$ have proposed an electronic structure theory in solution by using the molecular Ornstein-Zernike equation, which is referred as the MOZ-SCF method. In their treatment, the exchange repulsion/charge transfer interactions are incorporated for calculating the solute-solvent interactions. Unlike the former treatments, the MOZ-SCF has an advantage in describing the solvent distribution, which comes from the use of the integral equation theory. Utilization of the integral equation theory gives us the microscopic picture of solvation, and frees us from the sampling problem faced in the molecular simulation which is needed in the QM/MM method for liquid systems. We can readily evaluate the chemical properties of interest in a variety of thermodynamic states. However, the MOZ-SCF method has some trouble- 
some aspects in determination of the short-range solutesolvent interactions. The method requires $a b$ initio calculations for many configurations of solvents to fit the parameter for the short-range interactions; thereby, it costs a lot of computational time.

In this paper, we propose a new approach based on the integral equation theory of liquids which enables us to account the quantum nature in the short-range interaction between solute and solvent in solution. In order to describe the solute-solvent interaction, we apply a kind of modelpotential method based on the Hartree-Fock frozen density formulation, so that the interaction is described essentially in terms of the quantum chemistry. Combining the two theoretical frameworks, the model-potential method and the integral equation theory of liquids, we construct the electronic structure theory in solution. As the first step, we focus our attention on a system consisting of monatomic molecules, in which a solute is at infinite dilution in solvent.

The idea behind the model-potential method is to separate chemically active electrons from inactive ones in a molecule, and to project the inactive electrons onto active ones to construct a model potential or an effective Hamiltonian. The core-valence separation in atoms is the best-known example. In this case, the core electrons are projected onto the valence electrons. A mathematical device referred to as the "spectral representation" method has been used to facilitate the projection. For instance, the exchange interaction and/or Coulomb interaction between the core and valence electrons are represented in terms of an effective potential for the valence electrons by means of the spectral representation method. ${ }^{11,12}$ The method has been extended to a more general system in which molecules form a cluster. ${ }^{13-15}$ In the treatment, molecules in a cluster are classified into two parts: a molecule for which the electronic structure is explicitly calculated, and the rest, which are regarded as an "environment" to the molecule. The exchange and Coulomb interactions between the molecule and the environment are mapped into an effective Hamiltonian, or the "environmental potential," for the molecule. In the present paper, the environmental potential method ${ }^{14,15}$ is used for describing the quantum effect exerted on a solute from solvent molecules, in which both the exchange and Coulomb interaction are treated by means of the spectral representation technique.

The organization of this paper is as follows. In Sec. II, we review briefly the environmental potential method, and present a electronic structure theory in solution based on the method. In Sec. III, we show the results concerning the electronic and solvation structure of noble atoms, the neon and the argon, solvated in liquid neon. We also investigate the electronic structure of an excess electron in liquid helium. It should be noted that both the electronic and the solvation structure of these systems can not be treated consistently by most existing theories in which the quantum-mechanical effects between solute and solvent are ignored. Concluding remarks are given in Sec. IV along with an idea for the generalization of the present treatment to the molecular liquid systems.

\section{THEORY}

\section{A. Outline of environmental potential method}

In this section, we review briefly the environmental potential method. Although this method can be applied to the molecules consisting of many atoms, here we consider an atomic cluster for an illustrative purpose. The Fock operator of the total cluster system is expressed as follows:

$$
F^{\mathrm{tot}}=-\frac{1}{2} \Delta-\sum_{N} \frac{Z_{N}}{r}+\sum_{r, s}\left(2 J_{r s}-K_{r s}\right) \sum_{j} c_{r j} c_{s j},
$$

where $Z_{N}$ is the nuclear charge of nucleus $N, r$ is the distance between an electron and nucleus. $J_{r s}$ and $K_{r s}$ are the Coulomb and exchange integrals, respectively. $c_{r j}$ is the molecular orbital(MO) coefficient. Subscripts $r$ and $s$ indicate the atomic orbitals, and $j$ means the molecular orbital. We can divide the cluster into one central atom(cent) and environmental atoms(env). The Fock operator in Eq. (1) is rewritten as

$$
\begin{aligned}
F^{\mathrm{tot}}= & -\frac{1}{2} \Delta-\frac{Z_{\text {cent }}}{r}+\sum_{r, s}^{\text {on cent }}\left(2 J_{r s}-K_{r s}\right) \\
& \times \sum_{j} c_{r j} c_{s j}-\sum_{M}^{\text {env }} \frac{Z_{M}}{r}+\sum_{t, u}^{\text {Rest of all contribution }}\left(2 J_{t u}-K_{t u}\right) \\
& \times \sum_{j} c_{t j} c_{u j} .
\end{aligned}
$$

Here, we assume that the total environmental effects on the central atom are approximated by a linear combination of the potential fields of the environmental atoms, and that the relaxation of the environmental molecular orbitals due to the interaction with the central atom is negligible. On the basis of Eq. (2), the Fock operator for the central atom including the effects of the environmental atoms can be written as

$F \cong-\frac{1}{2} \Delta-\frac{Z_{\text {cent }}}{r}+\sum_{r, s}^{\text {on cent }}\left(2 J_{r s}-K_{r s}\right) \sum_{j} c_{r j} c_{s j}+\sum_{D}^{\text {env }} \bar{V}_{D}$,

where

$$
\bar{V}_{D}=\frac{-Z_{D}}{r}+\sum_{r, s}^{\text {on } D}\left(2 J_{r s}-K_{r s}\right) \sum_{j} c_{r j}^{\prime} c_{s j}^{\prime} .
$$

The prime indicates that the molecular orbital coefficients are determined for the isolated system. The element of the above Fock operator is written as follows:

$$
F_{p q} \cong H_{p q}+G_{p q}+\sum_{D}^{\text {env }}\left\langle\chi_{p}\left|\bar{V}_{D}\right| \chi_{q}\right\rangle,
$$

where

$$
\begin{aligned}
\left\langle\chi_{p}\left|\bar{V}_{D}\right| \chi_{q}\right\rangle= & \left\langle\chi_{p}\left|\frac{-Z_{D}}{r}\right| \chi_{q}\right\rangle \\
& +\sum_{r, s}^{\text {on } D} P_{r s}^{\prime}\left[\left(\chi_{p} \chi_{q} \mid \chi_{r} \chi_{s}\right)-\frac{1}{2}\left(\chi_{p} \chi_{s} \mid \chi_{r} \chi_{q}\right)\right] .
\end{aligned}
$$


$P_{r s}$ is the density matrix of the environmental atom. Note that $p$ and $q$ correspond to the atomic orbitals of the central atom. On the other hand, $r$ and $s$ indicate those of the environmental atoms. $H_{p q}$ and $G_{p q}$ are the one- and two-electron terms of the central atom, respectively. Next, we replace the operator $\bar{V}_{D}$ by an appropriate spectral representation

$$
\bar{V}_{D} \rightarrow \Omega_{D} \bar{V}_{D} \Omega_{D},
$$

where

$$
\Omega_{D}=\sum_{a, b}^{\text {on } D}\left|f_{a}\right\rangle\left(\mathbf{S}^{-1}\right)_{a b}\left\langle f_{b}\right| .
$$

$\left\{f_{a}\right\}$ is an arbitrary basis function set for the spectral representation and $\mathbf{S}$ is its overlap matrix. We obtain the Fock matrix

$$
F_{p q} \sim H_{p q}+G_{p q}+\sum_{D}^{\text {env }}\left\langle\chi_{p}\left|\Omega_{D} \bar{V}_{D} \Omega_{D}\right| \chi_{q}\right\rangle .
$$

The molecular orbitals of the environmental atoms should be prevented from collapsing into the space that the environmental electrons occupy due to the penetration of the molecular orbitals of the central atom. This problem can be remedied by adding the following projection operator, the so-called energy shift operator, to Eq. (9):

$$
\varrho_{D}=\sum_{j}^{\text {occ on } D} \sum_{r, s}^{\text {on } D}-n \epsilon_{j}^{\prime}\left|\chi_{r}\right\rangle c_{r j}^{\prime} c_{s j}^{\prime}\left\langle\chi_{s}\right|,
$$

where $\epsilon_{j}^{\prime}$ is the $j$ th molecular orbital energy of the isolated atom $D$, and $n$ is an arbitrary positive parameter. The energy shift operator simulates the effect of the Pauli exclusion principle and maintains the strong orthogonality of the molecular orbitals between central and environmental atoms. ${ }^{16}$ In the present work, the value of $n$ is determined so as to reproduce the results obtained by all-electron calculation, as is explained in the next section.

Finally, we obtain the approximated Fock matrix with the environmental effect

$$
F_{p q}=H_{p q}+G_{p q}+\sum_{D}^{\text {env }}\left\langle\chi_{p}\left|V_{D}\right| \chi_{q}\right\rangle,
$$

where

$$
\begin{aligned}
\left\langle\chi_{p}\left|V_{D}\right| \chi_{q}\right\rangle= & \left\langle\chi_{p}\left|\Omega_{D}\left\{\bar{V}_{D}+\varrho_{D}\right\} \Omega_{D}\right| \chi_{q}\right\rangle \\
= & \sum_{a, b, c, d}^{\text {on } D}\left\langle\chi_{p} \mid f_{a}\right\rangle\left(\mathbf{S}^{-1}\right)_{a b} \\
& \times\left\langle f_{b}\right|\left\{\frac{-Z_{D}}{r}+\sum_{j}{ }^{\text {on } D}\left(2 J_{j}-K_{j}\right)\right. \\
& \left.+\varrho_{D}\right\}\left|f_{c}\right\rangle\left(\mathbf{S}^{-1}\right)_{c d}\left\langle f_{d} \mid \chi_{q}\right\rangle .
\end{aligned}
$$

$V_{D}$ is the environmental potential exerted on the central atom from the environmental atom $D$. It should be noted that the nuclear repulsion term between the central and environmental atoms does not appear in the total Hamiltonian in the present system. It is assumed that the nuclear charges of the environmental atoms are perfectly shielded by the environmental electrons as is in case of the model core potential. ${ }^{17}$

\section{B. Formulation of an electronic structure of molecules in solution}

In the present section, we apply the environmental potential method to formulate a new theory for the electronic structure of a molecule in solution. Here, we separate the solute from solvent, and regard the latter as an environment to the former in the spirit of the environmental potential method. For describing the liquid structure, the integral equation theory is used in the present work. As the first step, we focus our attention on a system consisting of monatomic molecules, in which a solute is at infinite dilution in solvent. We also assume that the system is homogeneous and isotropic.

The interaction potential $u_{u v}(R)$ between solute and solvent separated by the distance $R$ is described by using the environmental potential as follows:

$$
\begin{aligned}
u_{u v}(R)= & 2 \sum_{i}\left\langle\varphi_{i}\left|V_{\text {solv }}\right| \varphi_{i}\right\rangle \\
= & \sum_{p, q} \sum_{a, b, c, d}^{\text {on solv }} P_{p q}\left\langle\chi_{p} \mid f_{a}\right\rangle\left(\mathbf{S}^{-1}\right)_{a b} \\
& \times\left\langle f_{b}\right|\left\{\frac{-Z}{r}+\sum_{j}^{\text {on solv }}\left(2 J_{j}-K_{j}\right)\right. \\
& \left.+\varrho_{\text {solv }}\right\}\left|f_{c}\right\rangle\left(\mathbf{S}^{-1}\right)_{c d}\left\langle f_{d} \mid \chi_{q}\right\rangle .
\end{aligned}
$$

$\varphi_{i}$ are the molecular orbitals of solute, which are expressed as a linear combination of the atomic orbitals $\chi_{p} . r$ is the separation between an electron and a solvent nucleus which has the charge $Z . J_{j}$ and $K_{j}$ are the Coulomb and exchange operator, respectively. $\varrho$ is the shift operator. $\left\{f_{a}\right\}$ is the spectral-representation basis set and $\mathbf{S}$ is its overlap matrix. Note that $\left\{f_{a}\right\}$ has the same origin with the solvent nucleus. The overlap matrix element $\left\langle\chi_{p} \mid f_{a}\right\rangle$ is a function of $\mathbf{R}$ $=(R, \theta, \phi)$ originated from solute. The problem associated with the integration over the angular space is explained in the Appendix.

As in the case of the RISM-SCF variational method, ${ }^{6}$ we derive the solvated Fock operator by the variation of the free-energy $A$ of the solute-solvent system. The free-energy $A$ is given as the sum of the solute electronic energy $E_{\text {solute }}$ and the excess chemical potential due to solute-solvent interactions $\Delta \mu$

$$
A=E_{\text {solute }}+\Delta \mu \text {. }
$$

For evaluating $E_{\text {solute }}$, various types of $a b$ initio electronic structure theory, such as multiconfigurational SCF and configuration interaction, can be applied. Here, we simply adopt the Hartree-Fock (HF) method for $E_{\text {solute, }}$, and the excess chemical potential derived from the OZ/HNC equation is adopted 


$$
\begin{aligned}
E_{\text {solute }}= & 2 \sum_{i}\left\langle\varphi_{i}|h| \varphi_{i}\right\rangle+\sum_{i, j}\left(2\left\langle\varphi_{i} \varphi_{i} \mid \varphi_{j} \varphi_{j}\right\rangle-\left\langle\varphi_{i} \varphi_{j} \mid \varphi_{j} \varphi_{i}\right\rangle\right) \\
& +E_{\text {nuc }}, \\
\Delta \mu=- & \frac{\rho}{\beta} \int\left\{\exp \left[-\beta u_{u v}(R)+t_{u v}(R)\right]-1-t_{u v}(R)\right. \\
& \left.-h_{u v}(R) t_{u v}(R)+\frac{1}{2} h_{u v}(R)^{2}\right\} d \mathbf{R} \\
- & \frac{1}{\beta} \int\left\{-c_{u v}(R) h_{u v}(R)+\frac{1}{2} c_{u v}(R) c_{u v}(R) * \chi_{v v}\right\} d \mathbf{R} .
\end{aligned}
$$

In the above expression, $h$ is the one-electron operator. $\beta$ is equal to $1 / k_{B} T$, where $k_{B}$ and $T$ are the Boltzmann constant and temperature, respectively. $\rho$ is the density of solvent. $c_{u v}$ and $h_{u v}$ are the direct and total correlation functions, respectively. $t_{u v}$ is equal to $h_{u v}-c_{u v} \cdot \chi_{v v}$ is the pure solvent site density pair correlation functions. The quantity $A$ can be regarded as a functional of the functions $h_{u v}, c_{u v}, t_{u v}$, and $\varphi_{i}$. We define $I$ with the constrains to the orthonormality of the molecular orbitals as

$$
I=A\left[h_{u v}, c_{u v}, t_{u v}, \varphi\right]-\sum_{i, m} \epsilon_{i m}\left(\left\langle\varphi_{i} \mid \varphi_{m}\right\rangle-\delta_{i m}\right) .
$$

Variations with respect to the functions yield

$$
\begin{aligned}
\delta I= & 2 \sum_{i}\left\langle\delta \varphi_{i}|h| \varphi_{i}\right\rangle+2 \sum_{i}\left\langle\delta \varphi_{i}\left|\sum_{j}\left(2 J_{j}-K_{j}\right)\right| \varphi_{i}\right\rangle \\
& -\rho \int 2 \sum_{i}\left\langle\delta \varphi_{i}\left|V_{\text {solv }}\right| \varphi_{i}\right\rangle \exp \left[-\beta u_{u v}(R)\right. \\
& \left.+t_{u v}(R)\right] d \mathbf{R}-\frac{\rho}{\beta} \int\left(\exp \left[-\beta u_{u v}(R)+t_{u v}(R)\right]\right. \\
& \left.-h_{u v}(R)-1\right) \delta t_{u v} d \mathbf{R}-\frac{\rho}{\beta} \int\left(-t_{u v}(R)+h_{u v}(R)\right. \\
& \left.-c_{u v}(R)\right) \delta h_{u v} d \mathbf{R}-\frac{1}{\beta} \int\left(h_{u v}(R)\right. \\
& \left.+c_{u v}(R) * \chi_{v v}\right) \delta c_{u v} d \mathbf{R}-\sum_{i, m} \epsilon_{i m}\left\langle\delta \varphi_{i} \mid \varphi_{m}\right\rangle .
\end{aligned}
$$

The fourth, fifth, and sixth terms give a set of the OZ/HNC equations. One can eventually obtain the expression for the solvated Fock matrix element

$$
F_{p q}=H_{p q}+G_{p q}+\rho \int\left\langle\chi_{p}\left|V_{\text {solv }}\right| \chi_{q}\right\rangle g_{u v}(R) d \mathbf{R},
$$

where $H_{p q}$ and $G_{p q}$ are the usual one- and two-electron terms, respectively. $g_{u v}(R)=h_{u v}(R)+1$ is the radial distribution function between solute and solvent.

In this way, we obtained a set of equations for the electronic structure in solution, i.e., the solute-solvent $\mathrm{OZ} / \mathrm{HNC}$ equation and the modified HF equation incorporating the quantum solvation effect. These equations are solved by a self-consistent procedure.

\section{RESULTS AND DISCUSSION}

In this section, we present some illustrative results obtained from the new theory presented in the previous section. The electronic and the solvation structure of a neon and an argon solvated in liquid neon, and an excess electron in liquid helium are calculated to demonstrate the validity of the present treatment. For the calculation of the density pair correlation function $\chi_{v v}$ in pure solvent, it is possible to use the environmental potential method as discussed above. However, in order to make the comparison of the present treatment with the classical approach easier, the radial distribution function between a solvent pair is determined by using the Lennard-Jones potential: $\sigma=2.75 \AA$ and $\epsilon=35.8 \mathrm{~K}$ are employed for the solvent neon site, ${ }^{18}$ and $\sigma=2.556 \AA$ and $\epsilon=10.2 \mathrm{~K}$ for the solvent helium..$^{19}$ Only the interaction between the solute and solvent is treated by using the environmental potential method. The electronic wave functions of the solute neon and argon atoms were calculated at the HF level with 6-311G basis set. For the basis set of the excess electron, we adopted the $6-311++\mathrm{G}^{* *}$ basis set for the hydrogen atom with the seven $s$-type functions and seven $p$-type functions. This extensively diffuse basis set was used for investigating the water dimer anion and trimer anion by Tsurusawa et al. ${ }^{20}$ The environmental potential was generated by projecting the reference electronic wave functions of the solvent on an arbitrary spectral-representation basis sets $\left\{f_{a}\right\}$. Although $\left\{f_{a}\right\}$ is desirable to be as complete as possible, a suitable choice of $\left\{f_{a}\right\}$, from the practical point of view, may be a set which is composed of primitive Gaussian type orbital (PGTO) decontracted from the atomic basis sets used in the reference calculation of solvent. Katsuki has applied the environmental potential method to the interaction between three ammonia molecules using the spectralrepresentation basis sets as mentioned above. The result obtained by the environmental potential method is in good agreement with that of the all-electron calculation. ${ }^{14}$

In this work, the reference electronic wave functions were calculated in the HF level with 6-311G basis set and the PGTO decontracted from 6-311G basis set is chosen as the spectral-representation basis set.

\section{A. Neon and argon solvated in liquid neon}

The radial distribution functions( $\mathrm{RDF}$ ) between the solute and solvent neons calculated by the present method are shown in Fig. 1. The RDFs obtained by the OZ/HNC equation using the LJ potential for the solute-solvent interaction are also presented in the figure. The LJ parameters used for the solute neon are the same as those used for calculating the pure solvent site density pair correlation function $\chi_{v v}$. The density dependences of the RDFs at 300 and at $100 \mathrm{~K}$ are shown in Figs. 1(a) and in 1(b), respectively. The density is the reduced density, $\rho^{*}=\rho \sigma^{3}$. The parameter $n$ of the shift operator is determined so as to reproduce best the interaction energy of the neon dimer obtained from an all-electron calculation with $6-311 \mathrm{G}$ basis set. The behavior of the repulsive part of the interaction at the distance of closest approach is our main concern in fitting the parameter, because that part is essential for determining the liquid structure. As shown in the figures, the present method reproduces reasonably well 

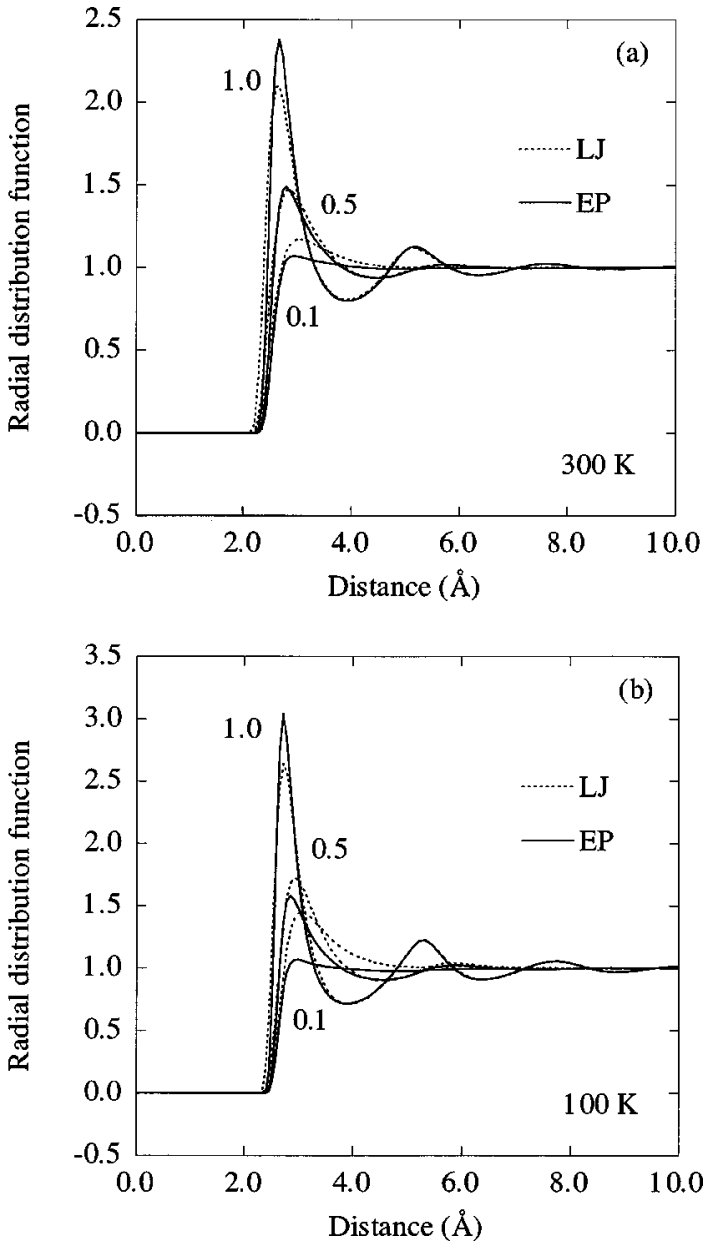

FIG. 1. The results for the radial distribution functions (RDFs) between the solute neon and solvent neon obtained by the two methods. LJ indicates the $\mathrm{OZ} / \mathrm{HNC}$ equation with the Lennard-Jones (LJ) interaction. EP indicates the present method using the environmental potential. (a) is the result at $300 \mathrm{~K}$, and (b) is at $100 \mathrm{~K}$.

the density and the temperature dependence of the RDFs obtained by using the LJ interaction potential: the positions of the contact between solute and solvent in the RDFs, and the decrease in the peak height around the solute neon with decreasing density. These results demonstrate capability of the present theory to predict the solvation structure at least in the qualitative level. From the quantitative view point, the disagreement becomes more conspicuous with decreasing density or cooling temperature. Generally speaking, the importance of the attractive forces between molecules for the liquid structure becomes larger at lower density as well as temperature. The environmental potential in the HF level cannot describe the attractive potential due to the dispersion force. Therefore, the solvation structure is more readily destructured due to decrease in density and temperature than that obtained by using the LJ interactions.

The results for RDFs between the solute argon and solvent neons are shown in Fig. 2 along with those from the $\mathrm{OZ} / \mathrm{HNC}$ equation using the $\mathrm{LJ}$ interactions. The $\mathrm{LJ}$ parameters used for solute argon are $\sigma=3.42 \AA$ and $\epsilon=124.03 \mathrm{~K}^{21}$ The parameter of the shift operator was determined by fitting the results with the all-electron calculation for the $\mathrm{Ar}-\mathrm{Ne}$ dimer. Although the results show the discrepancy between
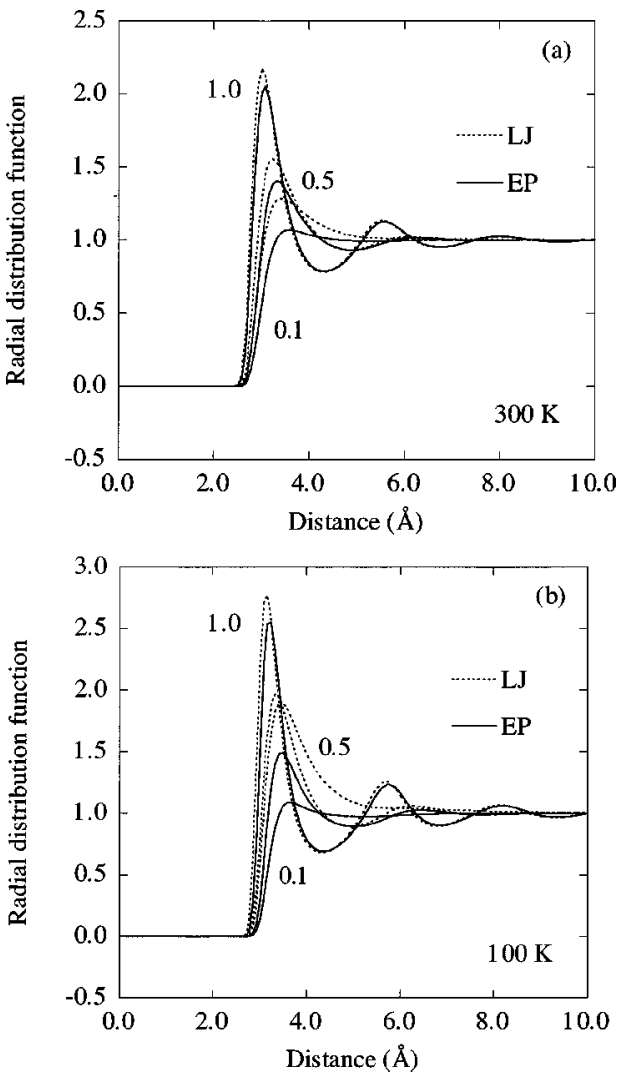

FIG. 2. The results for the RDFs between the solute argon and solvent neon obtained by the two methods. LJ indicates the OZ/HNC equation with the Lennard-Jones (LJ) interaction. EP indicates the present method using the environmental potential. (a) is the result at $300 \mathrm{~K}$, and (b) is at $100 \mathrm{~K}$.

the $\mathrm{LJ}$ potential and the present interactions in terms of the temperature and density dependences of RDF, similar to the previous case, the present treatment qualitatively reproduces the contact position between a solute argon and a solvent neon, and the density and temperature dependence of the RDFs.

In Fig. 3, the density dependence of the electronic spatial extent, which is an expected value of $r^{2}$, of a solute argon is

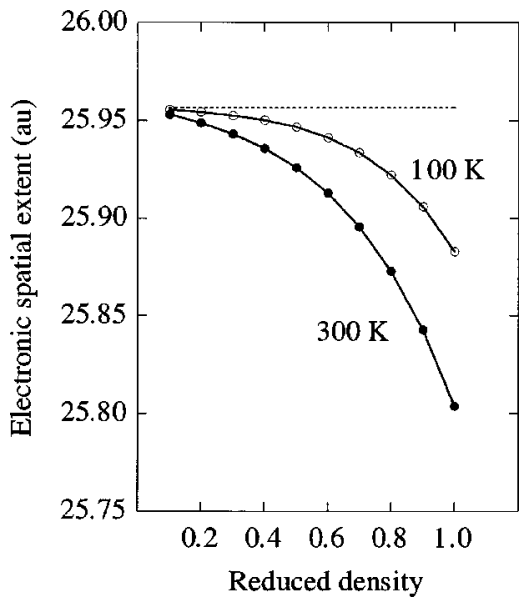

FIG. 3. The density dependence of the electronic spatial extents $\left\langle r^{2}\right\rangle$ of solute argon atom at $300 \mathrm{~K}$ (black circles) and at $100 \mathrm{~K}$ (open circles). The dotted line is the value in the gas phase. 


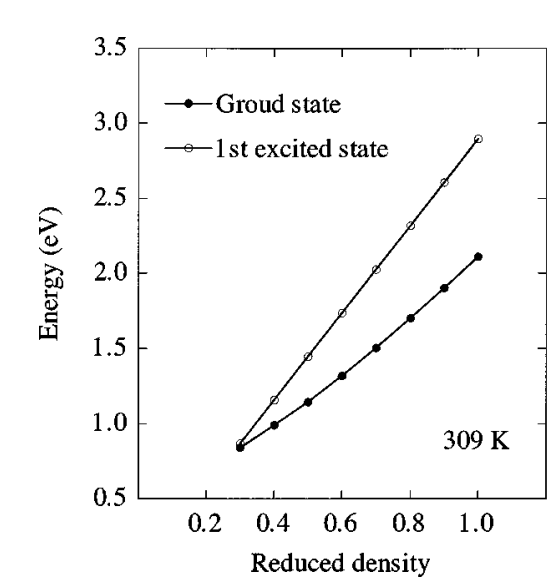

FIG. 4. The results for the ground and first-excited energies of an excess electron in liquid helium as a function of the solvent density.

shown along with that of an isolated argon atom in gas phase. Figure 3 is one of the principal results in this paper. It should be noted that if the LJ parameter is used as the solute-solvent interaction for this system, the constant value is obtained for the spatial extent through the various densities, which is same as in the gas phase. As can be readily seen from the figure, the spatial extent decreases with increasing density, that is, the short-range repulsive force exerted from solvents on the electronic structure of the solute argon increases. Additionally, the electronic extent of the solute argon is more sensitive to the density change at $300 \mathrm{~K}$ than that at $100 \mathrm{~K}$. This is due to the fact that the static thermal fluctuation of solvents increases with rising temperature. The temperature and density dependence of the electronic spatial extent of a neon atom also has same trend. Although an excess repulsive character of the interaction may appear in the behavior of $\left\langle r^{2}\right\rangle$ due to the absence of the attractive interaction such as dispersion force in the present treatment, these results are in accord with our intuition, strongly indicating that our attempt is robust.

\section{B. Excess electron in liquid helium}

The present method can also treat an excess electron solvated in liquids. Here, we calculate the electronic and solvation structure of an electron in liquid helium. The groundstate energy was obtained by the HF method, and the firstexcited state energy in the case of the vertical transition was estimated by the singly excited configuration interaction. The parameter of the shift operator was determined by fitting the result for the hydrogen-helium dimer to that of the corresponding all-electron calculation. It should be noted that we can only discuss, in the present stage, the localized electronic state of an excess electron where the splitting between the ground and excited states is always larger compared with thermal energies.

The energy of the ground and first-excited states along the $309 \mathrm{~K}$ isotherm is shown in Fig. 4 as a function of density. Note that the electronic structure of the ground state has the $s$ character, and that of the first-excited state has the $p$ character. Since the present theory assumes that the system is homogeneous and isotropic, the first-excited state with the $p$
TABLE I. The energy eigenvalues for the ground and first excited states of the excess electron at $309 \mathrm{~K}$ in eV.

\begin{tabular}{ccccc}
\hline \hline$\rho^{*}$ & 0.3 & 0.5 & 0.7 & 0.9 \\
\hline$E_{0}$ & 0.84 & 1.15 & 1.51 & 1.90 \\
$E_{1}$ & 0.87 & 1.45 & 2.03 & 2.61 \\
\hline \hline
\end{tabular}

character degenerates triply. It should be noted that the solvation structure is not reorganized for the first-excited state in this treatment, that is, the vertical transition. This condition would be reflected in the very diffuse character of the electronic spatial extent $\left\langle r^{2}\right\rangle$ of the first-excited state. In the present estimation, at $\rho^{*}=0.9$, for example, $\left\langle r^{2}\right\rangle$ of the ground state is 23 a.u. and that of the first-excited state is $1.3 \times 10^{6}$ a.u.

Some of the energy eigenvalues in Fig. 4 are listed in Table I. As the density gets higher, the energies go up. The energy of the excited state increases more rapidly than that of the ground state. The density dependence of the energetics is in accord with the previous theoretical works ${ }^{22-27}$ for an excess electron in liquid helium at the same temperature. The potential and kinetic energies of the ground state are also shown in Fig. 5 as a function of density, and the results obtained by the present treatment are in reasonable agreement with the previous works. ${ }^{22,23}$ These results demonstrate the robustness of the present treatment.

Figure 6 shows the solvation structure of an electron in liquid helium for the various fluid densities at $T=309 \mathrm{~K}$. As shown in the figure, the distance of the closest approach between the electron and helium changes largely with increasing density of solvent. If the particles in the system have the so-called "core" region, the distance of the closest approach will not change largely by the density change as shown in Figs. 1 and 2. This interesting behavior of solvation structure suggests that a solvated electron is "softer" than an electron consisting atom. However, the hardness of the excess electron in the liquid helium manifested in the solvation structure by the present treatment seems to be much less than what is obtained by the previous theoretical studies. ${ }^{22,27,28}$ Note that an electron is represented by an isomorphic ring polymer consisting of "beads" in the path integral

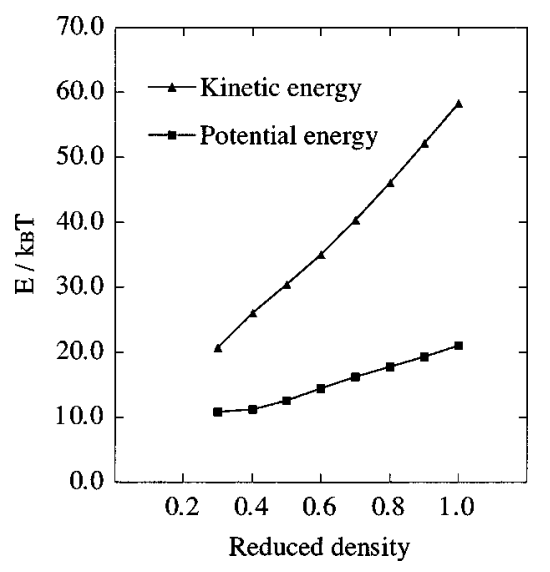

FIG. 5. The results for the potential and kinetic energies of the ground state of an excess electron in liquid helium as a function of the solvent density. 


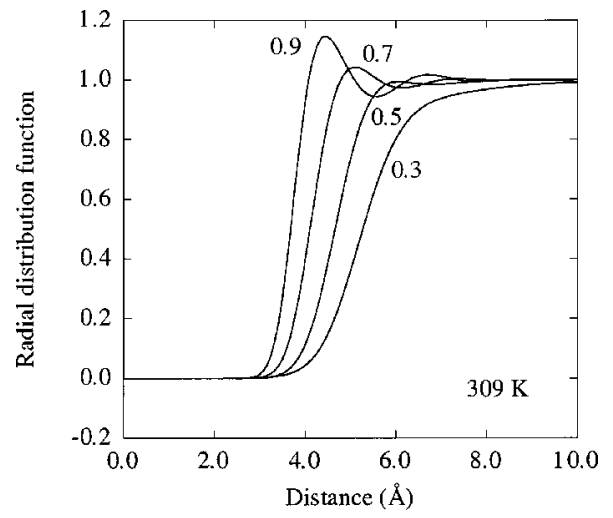

FIG. 6. The density dependence of the RDFs between the solute electron and solvent helium at $309 \mathrm{~K}$.

treatment, ${ }^{22,28}$ and that the present results should be compared with the RDF between the polymer center and solvent. According to the previous works, the distance of closest approach between the electron and helium has little dependence on the solvent density. This may be due to the repulsive character of the present potential. Since the parameter of the shift operator is fitted to the interaction between hydrogen and helium, the present potential is likely to be more repulsive than those in the previous works. It is also considered that the description of the present basis sets is insufficient for the excess electron and/or for the spectral representation. The pseudopotentials which are used in the previous theoretical studies mentioned above also have room for improvement. It is difficult at the present stage to conclude which solvation structure is more faithful to the real picture of the excess electron. Further experimental and theoretical studies are desired for elucidating the structure of solvated electrons as a function of the density.

\section{CONCLUDING REMARKS}

In the present paper, we have presented a new approach for the electronic structure of a molecule in solution, in which both the solute electronic structure and the solutesolvent interactions are treated based on the Hartree-Fock frozen density formulation. The spectral representation technique was employed to project the solvent environment onto the solute Hamiltonian in the same spirit with the environmental potential method. The integral equation theory of liquids is employed to calculate the solvent distribution around solute. The electronic and solvation structure of neon and argon solvated in liquid neon, and the excess electron in liquid helium were investigated as illustrative applications of the new theory. The present method reproduced reasonably well the density and the temperature dependence of the $\mathrm{Ne}-\mathrm{Ne}$ and $\mathrm{Ar}-\mathrm{Ne}$ RDFs obtained by using the LJ interaction potential. The results for the density dependence of the electronic spatial extent $\left\langle r^{2}\right\rangle$ of the solute argon showed that the quantum-mechanical effects from the solvent is incorporated successfully and explicitly into the solute electronic structure, unlike the conventional electronic structure theory in solution. The energy eigenvalues for the ground and firstexcited states of the excess electron in liquid helium obtained by the present method were in accord with those obtained by the previous theoretical studies. These results demonstrate the robustness of the present method.

Extending the present treatment to the molecular liquid systems is important from a view point of chemistry. We employ the 3D-RISM theory for describing the solvation structure of molecular liquid systems, unlike the MOZ-SCF method, and construct the electronic structure theory in solution as in the case of the 3D-RISM/SCF theory. ${ }^{29}$ In order to incorporate the environmental potential method into the RISM theory, we have to prepare the site-site environmental potential of solvent molecule by using some approximations. A possible strategy for the site-site environmental potential, for example, is to project the quantum effect of solvent onto the spectral-representation basis sets originated from each site of solvent molecule, and to ignore the contribution of cross term between different sites on the solvent molecule. Such study is in progress in our laboratory.

It it is of great interest to improve the accuracy of the present potential, which concerns the choice of the shift operator, the basis set for the spectral representation, the solvent polarization, and charge transfer as well as the electron correlation between solute and solvent. Those are among our future plans.

\section{ACKNOWLEDGMENTS}

This work has been supported by the Grant in Aid for Scientific Research on Priority Areas "Molecular Physical Chemistry" (403-12042285) from the Ministry of Education, Science, Sports and Culture (MONBUKAGAKUSHO) in Japan. H.S. thanks Grant-in Aid for Encouragement of Young Scientists, the Japanese Ministry of Education, Science, Sports and Culture (MONBU SHO). The authors are grateful to Professor Eisaku Miyoshi of Kyushu University and Dr. Tomonari Sumi of Toyohashi University of Technology for their suggestive comments, invaluable discussions, and encouragement.

\section{APPENDIX: INTEGRATION OVER THE ANGULAR SPACE}

As was stated in the explanation of Eq. (13), $\Lambda_{p q}$ $\equiv\left\langle\chi_{p}\left|V^{\text {solv }}\right| \chi_{q}\right\rangle$ is the function of $\mathbf{R}$. In the present stage, we simply adopt the interpolation manner for evaluating $\Lambda_{p q}(R, \theta, \phi)$ as follows.

Considering the sphere with the radius $R$ and dividing the sphere into octants, each point on the spherical surface has the value of $\Lambda_{p q}(R, \theta, \phi)$. In the case of the first octant as shown in Fig. 7, for example, three vertices have the values of $\Lambda_{p q}(R, 0,0), \Lambda_{p q}(R, \pi / 2,0)$, and $\Lambda_{p q}(R, \pi / 2, \pi / 2)$. The values of $\Lambda_{p q}(R, \theta, \phi)$ in the first octant can be represented by using these three values as follows:

$$
\begin{aligned}
\Lambda_{p q}(R, \theta, \phi)= & \sin ^{2} \theta \cos ^{2} \phi \Lambda_{p q}(R, \pi / 2,0) \\
& +\sin ^{2} \theta \sin ^{2} \phi \Lambda_{p q}(R, \pi / 2, \pi / 2) \\
& +\cos ^{2} \theta \Lambda_{p q}(R, 0,0) .
\end{aligned}
$$

The rest of the octants can be evaluated in the same manner to get 


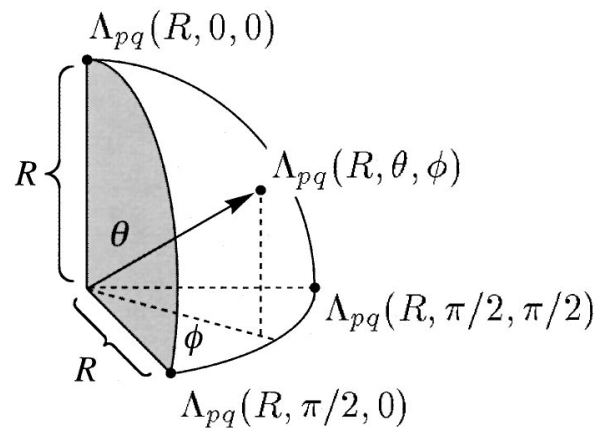

FIG. 7. $\Lambda_{p q}$ defined at grid points in an octant with the radius $R$.

$$
\begin{aligned}
\rho \int\left\langle\chi_{p}\left|V^{\mathrm{solv}}\right| \chi_{q}\right\rangle g_{u v}(R) d \mathbf{R} \\
=\rho \iiint \Lambda_{p q}(R, \theta, \phi) g_{u v}(R) R^{2} \sin \theta d R d \theta d \phi \\
=\rho \int \frac{2}{3} \pi\left[\Lambda_{p q}(R, 0,0)+\Lambda_{p q}(R, \pi / 2,0)\right. \\
\quad+\Lambda_{p q}(R, \pi / 2, \pi / 2)+\Lambda_{p q}(R, \pi, 0)+\Lambda_{p q}(R, \pi / 2, \pi) \\
\left.\quad+\Lambda_{p q}(R, \pi / 2,3 \pi / 2)\right] R^{2} g_{u v}(R) d R .
\end{aligned}
$$

By evaluating $\Lambda_{p q}$ at grid points in Eq. (A2), we can get the solvated Fock matrix of Eq. (19).

${ }^{1}$ J. Tomasi and M. Persico, Chem. Rev. 94, 2027 (1994).

${ }^{2}$ J. Gao, Acc. Chem. Res. 29, 298 (1996).
${ }^{3}$ C. J. Cramer and D. G. Truhlar, Chem. Rev. 99, 2161 (1999).

${ }^{4}$ S. Ten-no, F. Hirata, and S. Kato, Chem. Phys. Lett. 214, 391 (1993).

${ }^{5}$ S. Ten-no, F. Hirata, and S. Kato, J. Chem. Phys. 100, 7443 (1994).

${ }^{6}$ H. Sato, F. Hirata, and S. Kato, J. Chem. Phys. 105, 1546 (1996).

${ }^{7}$ C. Amovilli and B. Mennucci, J. Phys. Chem. B 101, 1051 (1997).

${ }^{8}$ P. N. Day, J. H. Jensen, M. S. Gordon, S. P. Webb, W. J. Stevens, M. Krauss, D. Garmer, H. Basch, and D. Cohen, J. Chem. Phys. 105, 1968 (1996).

${ }^{9}$ T. Wesolowski, R. P. Muller, and A. Warshel, J. Phys. Chem. 100, 15444 (1996).

${ }^{10}$ N. Yoshida and S. Kato, J. Chem. Phys. 113, 4974 (2000).

${ }^{11}$ S. Huzinaga, L. Seijo, Z. Barandiarán, and M. Klobukowski, J. Chem. Phys. 86, 2132 (1987).

${ }^{12}$ S. Katsuki and S. Huzinaga, Chem. Phys. Lett. 152, 203 (1988).

${ }^{13}$ Z. Barandiarán and L. Seijo, J. Chem. Phys. 89, 5739 (1988).

${ }^{14}$ S. Katsuki, Can. J. Chem. 70, 285 (1992).

${ }^{15}$ S. Katsuki, J. Chem. Phys. 98, 496 (1993).

${ }^{16}$ S. Huzinaga, J. Mol. Struct.: THEOCHEM 234, 51 (1991).

${ }^{17}$ S. Huzinaga, Can. J. Chem. 73, 619 (1995).

${ }^{18}$ J. O. Hirschfelder, C. F. Curtis, and R. B. Bird, Molecular Theory of Gases and Liquids (Wiley, New York, 1966).

${ }^{19}$ A. Michels and H. Wouters, Physica (Amsterdam) 8, 923 (1941).

${ }^{20}$ T. Tsurusawa and S. Iwata, Chem. Phys. Lett. 287, 553 (1998).

${ }^{21}$ P. W. Atkins, Physical Chemistry, 2nd ed. (Oxford University Press, Oxford, 1984).

${ }^{22}$ D. F. Coker, B. J. Berne, and D. Thirumalai, J. Chem. Phys. 86, 5689 (1987).

${ }^{23}$ D. Laria and D. Chandler, J. Chem. Phys. 87, 4088 (1987).

${ }^{24}$ D. F. Coker and B. J. Berne, J. Chem. Phys. 89, 2128 (1988).

${ }^{25}$ B. Boltjes, C. de Graaf, and R. P. H. Rettschnick, J. Chem. Phys. 97, 408 (1992).

${ }^{26}$ B. Space, D. F. Coker, Z. H. Liu, B. J. Berne, and G. Martyna, J. Chem. Phys. 97, 2002 (1992).

${ }^{27}$ J. Zhu and R. I. Cukier, J. Chem. Phys. 99, 1288 (1993).

${ }^{28}$ M. Sprik, M. L. Klein, and D. Chandler, J. Chem. Phys. 83, 3042 (1985).

${ }^{29}$ H. Sato, A. Kovalenko, and F. Hirata, J. Chem. Phys. 112, 9463 (2000). 